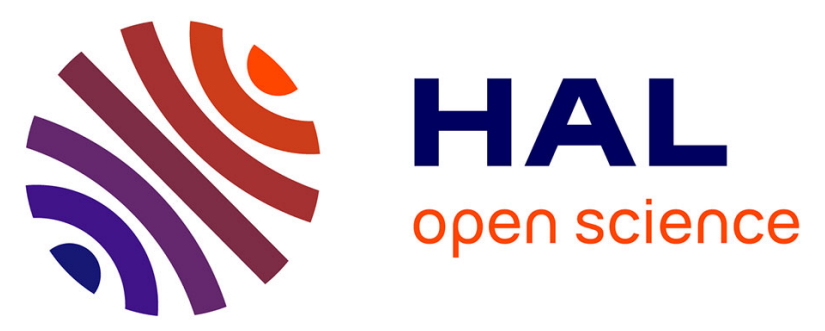

\title{
Linear to radial/azimuthal polarization converter in transmission using form birefringence in a segmented silicon grating manufactured by high productivity microelectronic technologies
}

Thomas Kampfe, Pierre Sixt, Denis Renaud, Armelle Lagrange, Fabrice

Perrin, Olivier Parriaux

\section{To cite this version:}

Thomas Kampfe, Pierre Sixt, Denis Renaud, Armelle Lagrange, Fabrice Perrin, et al.. Linear to radial/azimuthal polarization converter in transmission using form birefringence in a segmented silicon grating manufactured by high productivity microelectronic technologies. SPIE Photonics Europe, Micro-Optics Conference, Apr 2014, Brussels, Belgium. pp.91300W, 10.1117/12.2052156 . hal01020883

\section{HAL Id: hal-01020883 https://hal.science/hal-01020883}

Submitted on 8 Jul 2014

HAL is a multi-disciplinary open access archive for the deposit and dissemination of scientific research documents, whether they are published or not. The documents may come from teaching and research institutions in France or abroad, or from public or private research centers.
L'archive ouverte pluridisciplinaire HAL, est destinée au dépôt et à la diffusion de documents scientifiques de niveau recherche, publiés ou non, émanant des établissements d'enseignement et de recherche français ou étrangers, des laboratoires publics ou privés. 


\title{
Linear to radial/azimuthal polarization converter in transmission using form birefringence in a segmented silicon grating manufactured by high productivity microelectronic technologies
}

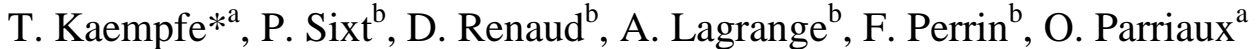 \\ ${ }^{a}$ Laboratoire Hubert Curien UMR CNRS 5516, Université de Lyon à St-Etienne, France; \\ ${ }^{\mathrm{b}}$ Laboratoire d'électronique et de technologie de l'information, CEA / Léti, 17 Rue des Martyrs, \\ 38054 Grenoble
}

\begin{abstract}
A polarization rotation is realized by subwavelength binary gratings, where the TE and TM round trip phases of the smallest grating modes are fixed to the smallest possible integer numbers of $2 \pi$ that allow a straight-through phase difference of $\pi$. This results in a subwavelength grating allowing to realize a half-wave element of almost $100 \%$ transmission. The principle is applied to a polarization transformation in the 1030-1064 $\mathrm{nm}$ wavelength range, using a segmented polarization rotating element converting a linearly polarized incidence to a radial or azimuthal polarization distribution. The elevated costs of such kind of polarization transformers based on assembled birefringent crystals are avoided by using mass-fabrication compatible silicon on insulator technology on a wafer scale. It shows the general potential of microelectronic technology, concerning the batch manufacturing of wavelength-scale diffractive, grating based elements for processing free space waves
\end{abstract}

Keywords: polarization transformation, subwavelength grating, effective index, radial polarization, azimuthal polarization, silicon on insulator

\section{INTRODUCTION}

Radial and azimuthal polarization is interesting for diverse applications, ranging from fundamental research to practical use in industrial fabrication, as detailed in several publications [1-3]. The creation of those types of polarization distribution can be done inside the laser resonator by a grating with circular symmetry placed on a multilayer mirror, where one polarization component leaks into the multilayer [4]. The resonator will then create a donut field profile as its fundamental mode, which is not necessarily compatible with complex high power laser architectures. Therefore, there is a need for extra-cavity polarization transformers, accepting a usual linearly polarized beam and transforming it into a radial or azimuthal polarization distribution (Fig. 1).

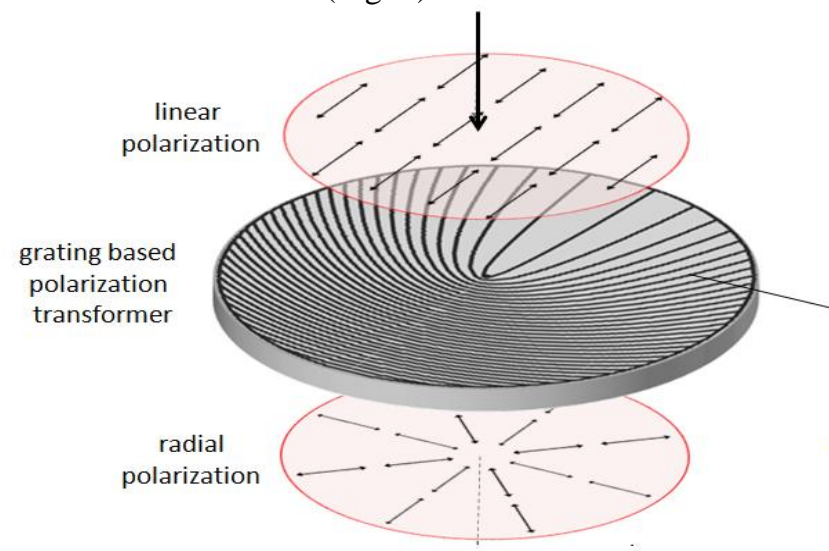

(a)

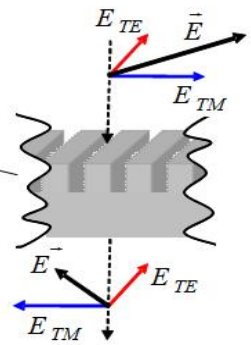

(b)

Figure 1. (a) Transformation of linear to radial polarization by a subwavelength grating. (b) the grating introduces a $\pi$-phase shift between TE and TM, leading to a mirroring of the E-field with respect to the local grating line direction 
A known solution is an assembly of angular segments of crystal quartz with half-wave thickness, employing a different orientation of the in-plane crystal axis per segment [5], which has the advantage of a high damage threshold, but is expensive and not mass fabrication compatible. The same principle can be realized also by using the form birefringence of a subwavelength binary grating of varying direction, etched into a high index substrate. However, the required small period and especially the large aspect ratio of the grooves [6] are a challenge for the fabrication technology, especially if the use of mass-fabrication compatible fabrication steps is envisaged. Additionally, a high transmission for both polarizations and the required $\pi$ phase difference cannot be optimally fulfilled simultaneously by such a structure. In this paper we describe a subwavelength grating achieving close to $100 \%$ transmission and the required phase difference, using a high index grating material on a low index substrate, offering the unique possibility of realizing this functionality with a reasonable aspect ratio of the grating and thus significantly relaxed fabrication technology requirements [7,8]. Using amorphous silicon in a silicon on insulator based lithographic fabrication chain, planar batch-technologies developed for microelectronics can be employed, envisioning inexpensive, high damage threshold polarization transforming elements.

\section{THEORY OF TE/TM PHASE OPTIMIZATION FOR REALIZING SMALL ASPECT RATIO $0^{\text {TH }}$ ORDER POLARIZATION TURNING TRANSMISSION GRATINGS}

The structure used for the polarization transformation is a high index binary grating on a low index substrate, whose parameters are chosen so that only the fundamental TE and TM grating mode are propagating, all higher grating modes being evanescent [7] (Fig. 2(b)).

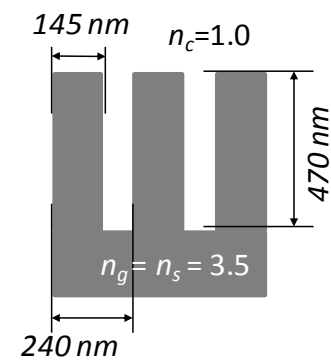

(a)

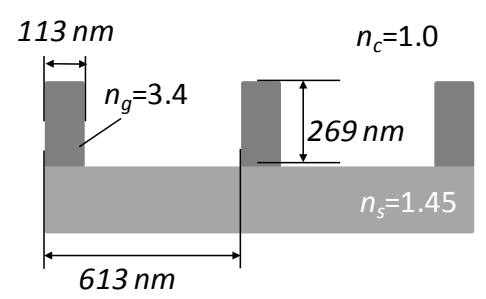

(b)

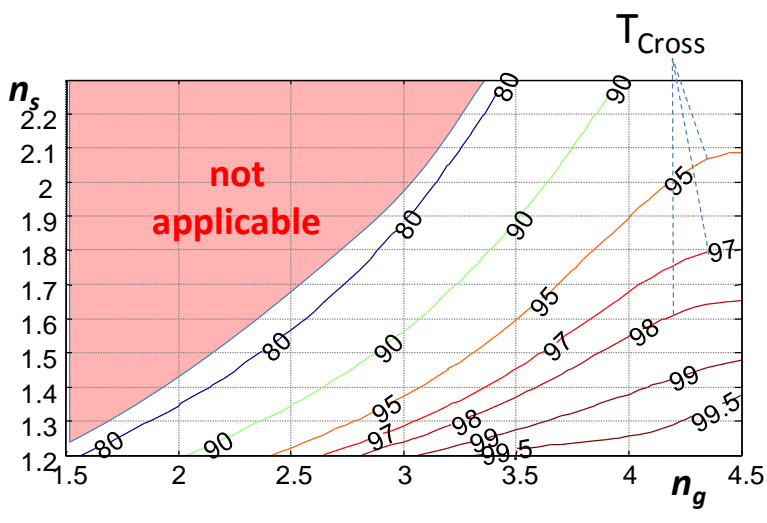

(c)

Figure 2. Polarization transforming grating creating a $\pi$-phase shift between TE and TM polarization, made by directly structuring the substrate (a) and by using a high index layer on a low index substrate (b). The region of applicability of the principle (b) for different substrate and grating materials is shown in (c) using Tcross (see text) as merit value.

Using a high index grating on a low index substrate has four advantages with respect to a grating etched directly into a high-index substrate (example from [6] shown in Fig. 2(a)). Firstly due to the larger period and much less severe aspect ratio of the grooves, the fabrication is significantly facilitated. Secondly, provided the parameters are chosen as detailed in [7] so that the effective index of the fundamental TM grating mode falls between substrate and cover, an almost $100 \%$ transmission of the TM polarization is possible as the grating layer represents for this polarization an antireflection layer between the cover and the substrate. Thirdly, an almost $100 \%$ transmission is possible for the equivalent TE transmission, since the Fabry-Perot resonator for the $\mathrm{TE}_{0}$ grating mode can be made symmetric, i.e. the effective index of this mode can be chosen larger than both air and substrate index. And finally, choosing a relatively large period changes the phases of the reflection/transmission coefficients, which are influencing the phase round-trip conditions of the equivalent TE and TM Fabry-Perots, leading to more design freedom and the possibility of fulfilling the TE Fabry-Perot $_{0}$ transmission resonance and the TE/TM $\pi$-phaseshift condition at the same time. Additionally, it can be shown that the use of the smallest possible integer numbers for the Fabry-Perot roundtrips to achieve transmission resonance becomes possible [7], explaining the mentioned lower aspect ratios (ridge aspect ratio $\approx 2$, groove aspect ratio $<1$, as compared to 4 
or more for both of those aspect ratios in a grating etched directly into a high index substrate). It is possible to explore the grating parameter space of substrate and grating index exhaustively in order to find all possible gratings realizing the described phase compensation scheme (Fig. 2(c)). As an easy merit value the transmission through crossed polarizers with the grating being oriented at $45^{\circ}\left(\mathrm{T}_{\text {cross }}\right)$ is used, since it will monotonically approach $100 \%$ if and only if the two conditions of $100 \%$ TE and TM transmission as well as a $\pi$ phaseshift between TE and TM are approached. As can be seen from Fig. 2(c) the higher the ridge index and the lower the substrate index, the better $\mathrm{T}_{\text {cross }}$ can be optimized, with a $\mathrm{n} \approx 3.5$ grating on $\mathrm{n} \approx 1.5$ substrate showing already $\mathrm{T}_{\text {cross }}=97 \%$, thus pointing to SOI technology as a viable solution.

\section{PTOTOTYPE FABRICATION BY DIRECT MASK-TRANSFER}

To demonstrate the feasibility of the proposed polarization transformer, several prototypes using a simplified laboratory technology chain were fabricated (Fig. 3), as detailed in [9], and explained on a basic level in the following.

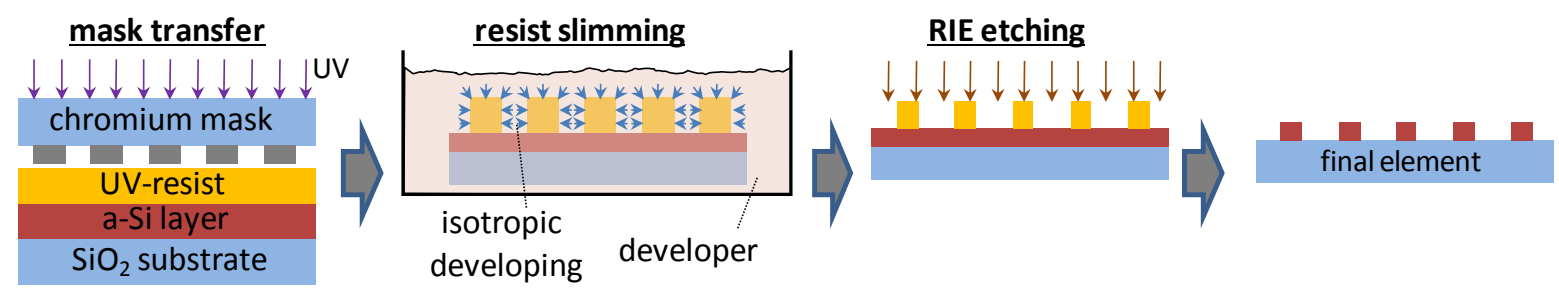

Figure 3. Left: Fabrication steps for a flexible production of laboratory prototype by direct mask-transfer for testing and optimization

We started by fabricating a standard chromium mask by e-beam writing. This mask was used in a hard-contact setup and transferred photolithographically into a resist layer to define the $596 \mathrm{~nm}$ period grating. Since the finally desired line width was too small to be realized directly in this step, it was reduce by wet-chemical thinning in order to end up with a ridge width of about $100 \mathrm{~nm}$. Reactive ion etching was then applied to transfer the resist profile into the amorphous silicon layer down to the quartz surface. An SEM image of a slice of the grating cut out by a focused ion beam machine (FIB), measured in transmission, is shown in Fig. 4(a), and the whole element under white light illumination can be seen in Fig. 4(b). The desired grating parameters of period $=616 \mathrm{~nm}$, ridge width $=109 \mathrm{~nm}$ and grating height $=250 \mathrm{~nm}$ were realized with an error of some nanometers only. The principal applicability of such elements in laser machine processing was demonstrated in [10].

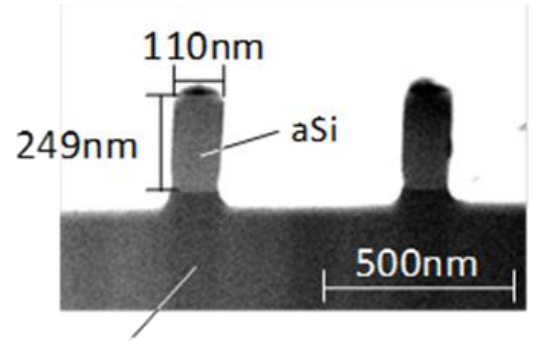

$\mathrm{SiO} 2$ substrate

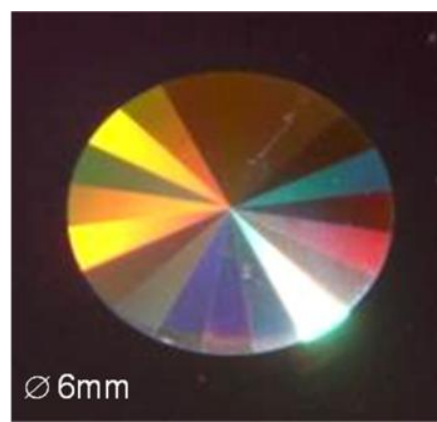

(b)

Figure 4. (a) SEM of the cross-section of the grating (extracted by FIB machining) of the laboratory prototype of the polarization transformer, (b) photograph of the prototype under white light 


\section{WAFER-SCALE, MASS-FABRICATION COMPATIBLE, SILICON ON INSULATOR BASED FABRICATION PROCESS}

A considerable advantage of the proposed approach of realizing grating based polarization transforming elements is its compatibility with batch processing technologies established for fabricating wafer-scale micro-electronics, namely the lithographic process of structuring a silicon layer placed on insulating substrates (silicon on insulator, SOI). We used the same design as in the case of the laboratory prototype, whose parameters were slightly adapted to period $=613 \mathrm{~nm}$, ridge width $=113 \mathrm{~nm}$ and grating height $=269 \mathrm{~nm}$ in order to account for the slightly different refractive index of the available deposited silicon of $n=3.4$. The process steps are depicted in Fig. 5.

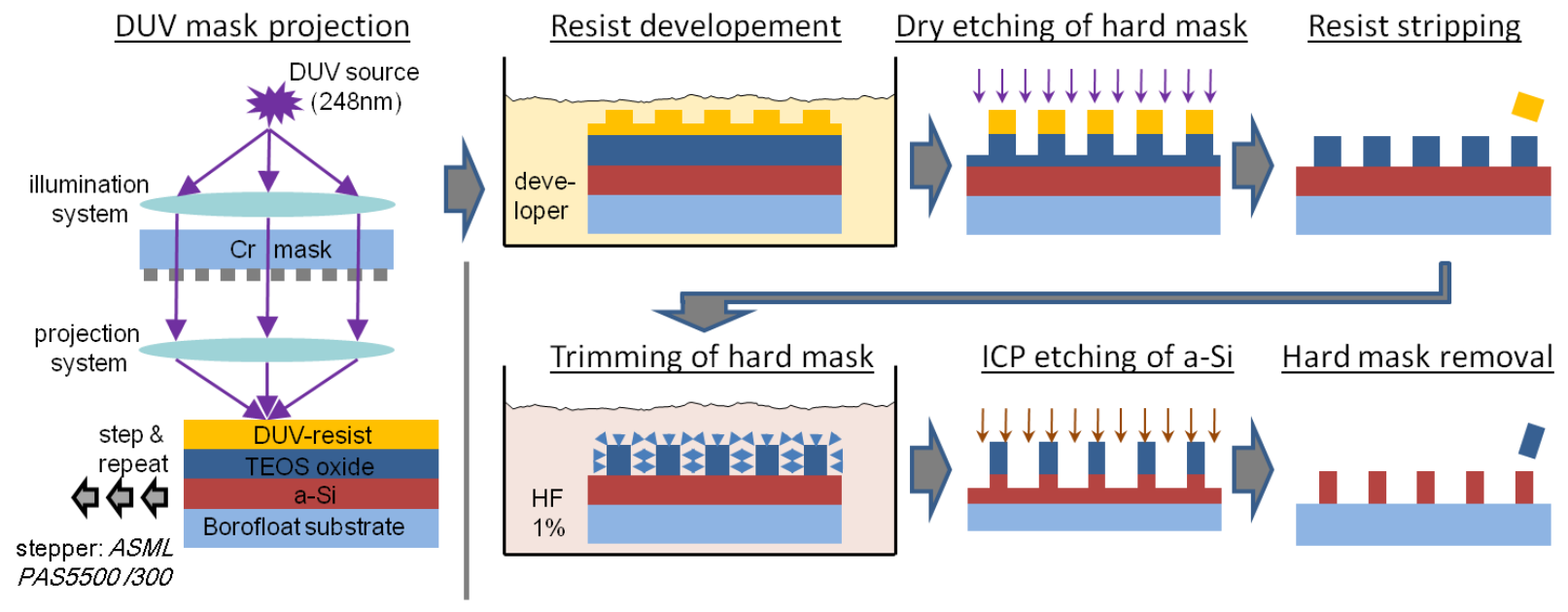

Figure 5: fabrication steps of step and repeat, 8" wafer-scale, mass-fabrication compatible lithography and etching process

We start by a deposition of an amorphous silicon layer by the well developed process of plasma enhanced physical vapor deposition (PECVD), known from amorphous silicon solar cells production, with a thickness equal to the desired grating height. Onto the silicon we deposited a 100nm thick tetraethylorthosilicate (TEOS) hard-mask layer. Subsequently, a resist layer (JSR M78Y) of $570 \mathrm{~nm}$ thickness as well as a bottom anti-reflective coating (BARC) of 50nm were applied onto the hard mask layer. The grating layout was realized as a chromium reticle with a scaling of 4 by standard e-beam lithography. This reticle was then projected by means of a $248 \mathrm{~nm} \mathrm{KrF}$ stepper (ASML PAS5500/300) with a 4x reduction factor onto the resist layer, creating 118 single elements per wafer. The resist layer was developed and subsequently transferred into the BARC and the hard mask layer by a inductively coupled plasma etching processes (ICP), using a $\mathrm{CF}_{4} / \mathrm{O}_{2} / \mathrm{Ar}$ mixture for the $\mathrm{BARC}$ etching and $\mathrm{C}_{4} \mathrm{~F}_{8} / \mathrm{O}_{2} / \mathrm{CO} / \mathrm{Ar}$ mixture for the transfer into the hard mask. Since the ridge width of the polarization transformer is too small to be realized directly, a wet-etching process using a $1 \% \mathrm{HF}$ solution is applied reducing the ridges isotropically by $40 \mathrm{~nm}$ in order to trim the line width down to the required $113 \mathrm{~nm}$. Finally, the hard-mask is transferred to the amorphous silicon layer in a highly selective ICP etching process using a $\mathrm{Cl}_{2} / \mathrm{HBr}$ mixture. The remnants of the hard mask, having a height of about 30nm, are subsequently removed via a $\mathrm{C}_{4} \mathrm{~F}_{8} / \mathrm{O}_{2} / \mathrm{CO} / \mathrm{Ar}$ ICP etching, where the length of the step is optimized in order to completely remove the remaining mask. The resulting gratings were analyzed in a FIB, showing a clean line profile (Fig. 6) with some deviations from the design: The height of the grating is smaller than the design, which is due to the removal process of the hard mask, that was slightly too long. A considerable overetch into the substrate of about 40nm can be seen, which is due to the hard mask removal being a non-selective process, i.e. attacking also the substrate. Finally the shape of the upper part of the lines is slightly trapezoidal, which is a sign for beginning mask degradation during the aSi-etching. All of those effects can be reduced by carefully optimizing the process. The effects of the changed grating parameters on the optical functionality will be detailed in the following chapter. All mentioned deviations are to be expected as part of the calibration of the SOI process chain, since the range of the parameters are different from better controlled microelectronics applications. The errors will be significantly reduced in further optimization steps. 


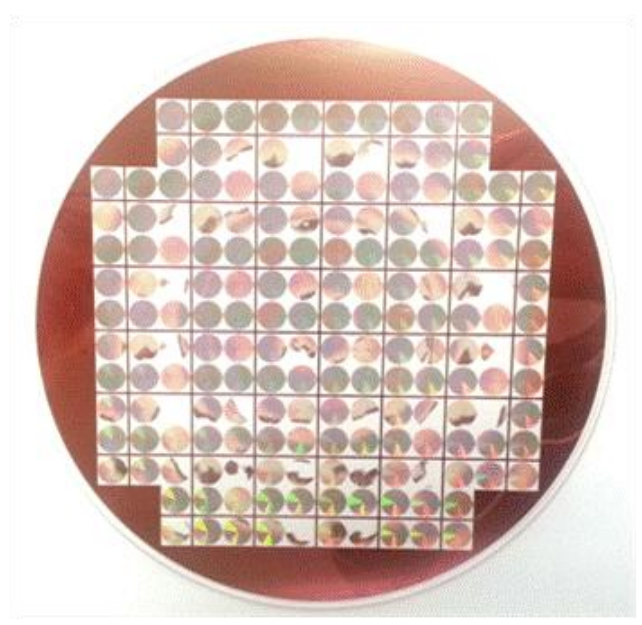

(a)
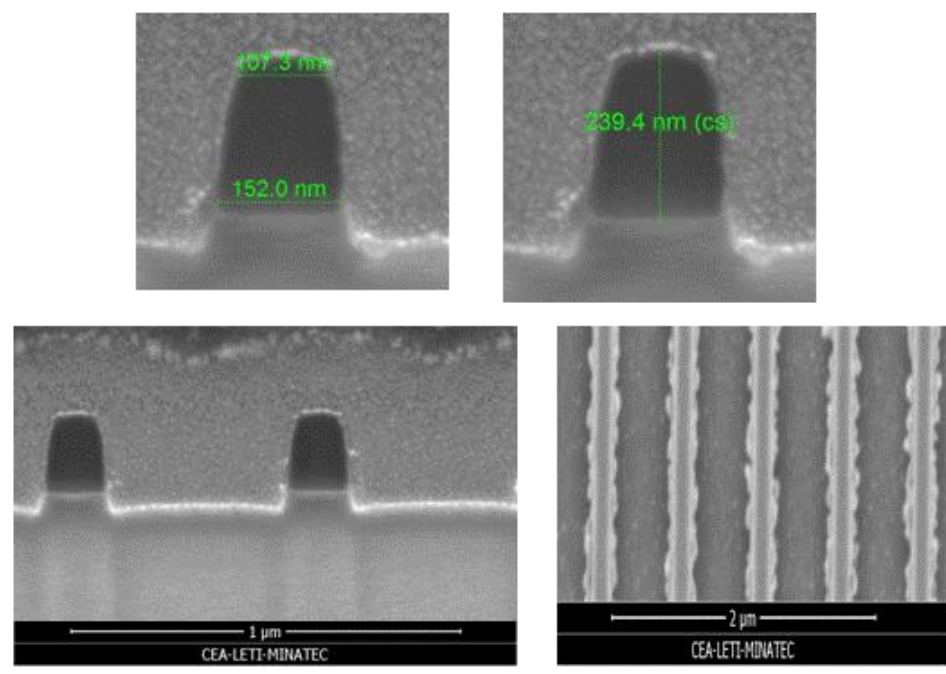

(b)

Figure 6: (a) photograph of a fabricated 8" wafer with 118 polarization transforming elements, (b) SEM images of the grating lines and their cross-section prepared by FIB

\section{CHARACTERIZATION AND OPTICAL TESTING}

The optical characterization of the elements was done using a collimated Nd:Yag laser with 1064nm wavelength (Fig. 7 (a)). The beam diameter was reduced via a telescope to $0.2 \mathrm{~mm}$ diameter in order to be able to measure the outer parts of the angular grating segments separately, in order to measure the transmission through crossed polarizers with the grating at $45^{\circ}$ ( see section 2 ).
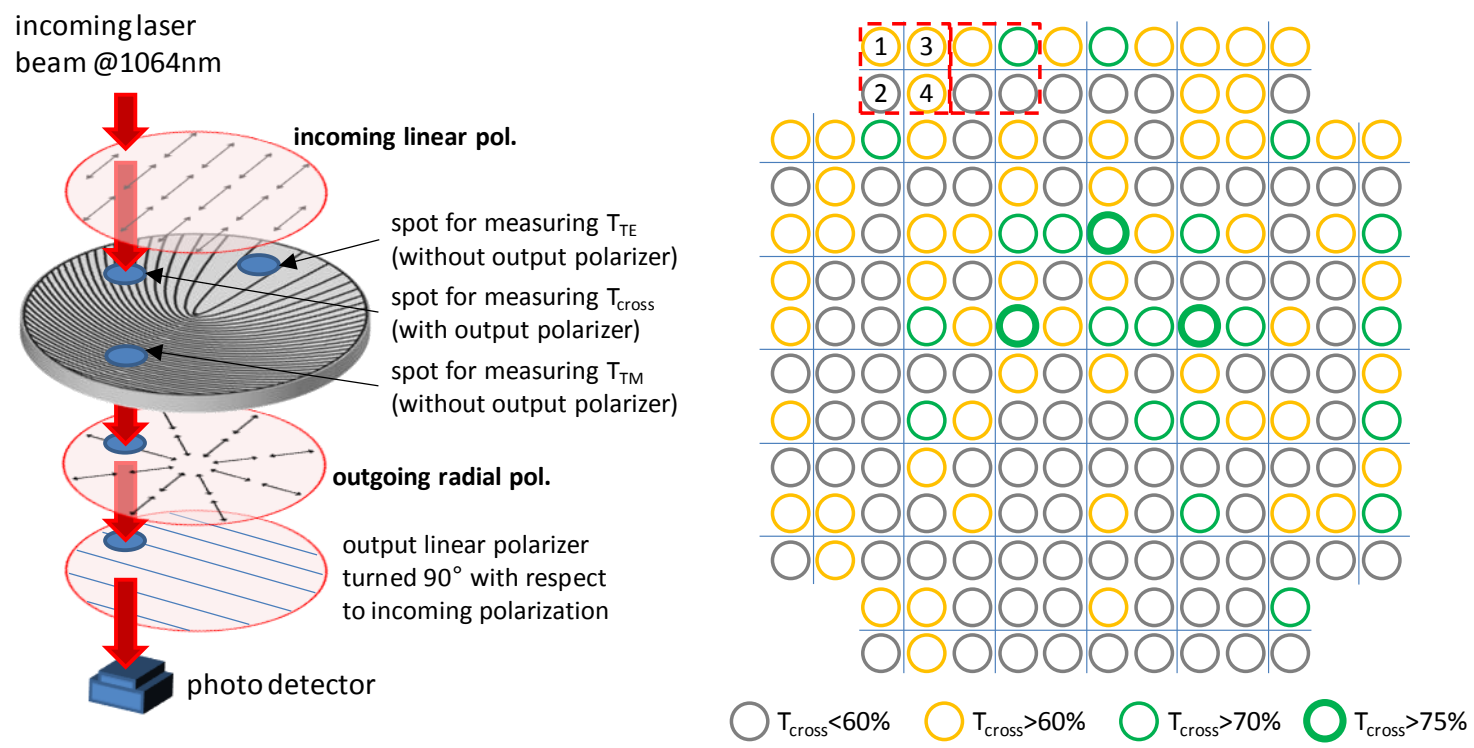

Figure 7. (a) Optical measurement setup for determining $\mathrm{T}_{\text {cross }}, \mathrm{T}_{\mathrm{TE}}$ and $\mathrm{T}_{\mathrm{TM}}$ (definition see text), (b) results for Tcross of a 8 " wafer with 118 elements (details of the element layout: see text). 
For the laboratory prototypes, a transmission of more than $97 \%$ for both polarizations could be measured. $\mathrm{T}_{\text {cross }}$ reached in some segments a maximum value of $95 \%$, with a theoretical maximum of $96.2 \%$. The average over all segments of $\mathrm{T}_{\text {cross }}$ was measured to be $90 \%$. The lower transmission in several sectors can be attributed to slight differences in the realized grating parameters, as well as to scattering due to a remaining line roughness that depends on the local orientation of the lines due to their definition in a shape-base e-beam machine. The efficient creation of radially polarized light was demonstrated by analyzing the intensity distribution of a widened beam (adapted to the element size of $6 \mathrm{~mm}$ ) which after passing through element was filtered by an additional linear polarizer. The output intensity distribution shows the typical butterfly shape, indicating the presence of radial polarization (Fig. 4(b) ). First tests to determine the laser flux resistance were made in a setup with a femtosecond Yb:YAG laser, leading to the main conclusion that the damage threshold of the polarization transformer in this case was close to the operation conditions of this machine [10], proving that an operation without additional beam diameter expansion is feasible.

The measurement of $\mathrm{T}_{\text {cross }}$ for a wafer of 118 elements fabricated by the process depicted in Fig. 5 (a) is shown in Fig. 7 (b). The layout in the $\mathrm{Cr}$ reticle was chosen to realize a variation of the line width in order to tune the process. In order to do this the line width in the Cr reticle for the 4 gratings arranged in a $2 \times 2$ layout as shown in the upper left corner by the dotted square in Fig. 7 (b) were chosen to be 180, 200, 220 and 240nm for the elements 1 to 4 respectively, thus allowing to find a grating with the correct line width of $113 \mathrm{~nm}$ after completing all process steps. This $2 \times 2$ layout was repeated over the whole wafer without additional changes in order to judge the overall homogeneity of the process. The results shown in Fig. 7 (b) lead to the following conclusions:

Firstly the polarization transformation could be verified but with a significantly lower transmission than in the case of the laboratory prototype. The $\mathrm{Cr}$ reticle grating line width which is best adapted is found to be $220 \mathrm{~nm}$ in quadrant 3 . Additionally the elements situated more towards the center of the wafer show a better performance. The FIB image of one of those elements is shown in Fig. 6 (b). A simulation made with the measured line width, grating height and overetch, including the trapezoidal line shape at the top, resulted in a theoretical value for $\mathrm{T}_{\text {cross }}$ of $82 \%$, while the measurement for this element remained at about Tcross $=76 \%$. The difference is probably caused mainly by scattering losses due to particles sticking to the lines (see SEM top view in Fig. 6 (b)).

Secondly there is a systematic variation over the wafer were the best elements are found in the middle. This is confirmed by the photograph in Fig. 6 (a) where a circular region of defective elements shows that there is some global variation of the process parameters, most probably connected to the layer deposition processes.

\section{CONCLUSION}

We demonstrated the design, fabrication and application of polarization transformers based on subwavelength gratings, made in a hydrogenated amorphous silicon layer on a low index substrate. We showed that the use of a low index substrate provides substantial advantages in comparison with a grating directly etched into the substrate. The optical functionality was demonstrated showing a good agreement with the theoretical calculations. To demonstrate the compatibility with mass fabrication technology several 8" wafers carrying 118 elements were fabricated, showing a less optimal optical performance due to deviation of the shape and parameters of the grating lines, caused by a not yet sufficiently optimized process chain. Beyond the realization of this particular polarization-rotating element, the presented work aims at evaluating what microelectronic technology can do for the batch manufacturing of diffractive optical elements with wavelength scale features for the processing of free space waves.

\section{REFERENCES}

[1] R. Weber, A. Michalowski, M. A. Ahmed, V. Onuseit, V. Rominger, M. Kraus, and T. Grafal, "Effects of Radial and Tangential Polarization in Laser Material Processing," Physics Procedia 12, pp. 21-30 (2011)

[2] T. Nieminen, N. Heckenberg, and H. Rubinsztein-Dunlop, "Forces in optical tweezers with radially and azimuthally polarized trapping beams," Opt. Lett. 33, 122-124 (2008).

[3] R. Dorn, S. Quabis, and G. Leuchs, "Sharper Focus for a Radially Polarized Light Beam” Phys. Rev. Lett. 91, 233901 (2003)

[4] M. A. Ahmed, J. Schulz, A. Voss, O. Parriaux, J. C. Pommier, and T. Graf, "Radially polarized $3 \mathrm{~kW}$ beam from a CO2 laser with an intracavity resonant grating mirror," Opt. Lett. 32, 1824-1826 (2007) 
[5] M. Kraus, M. A. Ahmed, A. Michalowski, A. Voss, R. Weber, and T. Graf, "Microdrilling in steel using ultrashort pulsed laser beams with radial and azimuthal polarization," Opt. Express 18 , pp. 22305-22313 (2010)

[6] G. M. Lerman and U. Levy, "Generation of a radially polarized light beam using space-variant subwavelength gratings at 1064 nm," Opt. Lett. 33, 2782-2784 (2008)

[7] T. Kämpfe and O. Parriaux, "Depth-minimized, large period half-wave corrugation for linear to radial and azimuthal polarization transformation by grating-mode phase management," J. Opt. Soc. Am. A 28, 2235-2242 (2011).

[8] O. Parriaux, T. Kämpfe, „Planar grating polarization transformer“, EP 2530499 A1 (2012)

[9] T. Kaempfe, S. Tonchev, G. Gomard, C. Seassal and O. Parriaux, "Hydrogenated amorphous silicon microstructuring for 0th order polarization elements at $1.0 \mu \mathrm{m}-1.1 \mu \mathrm{m}$ wavelength," IEEE Photonics Journal 3 , 1142-1148 (2011).

[10] R. Torres, T. Kaempfe, M. Delaigue, O. Parriaux, C. Hönninger, J. Lopez, R. Kling and E. Mottay, "Influence of laser beam polarization on laser micro-maching," 13th International Symposium on Laser Precision Microfabrication (2012). 